\title{
Restauração passiva em pastagens abandonadas a partir de núcleos de vegetação na Mata Atlântica, Brasil
}

\author{
Passive restoration of abandoned pastures from the nuclei of vegetation in the \\ Atlantic Rainforest, Brazil
}

\section{Pollyanna Rodrigues de O. dos Santos CoutinhoI, Ricardo Valcarcel" ${ }^{\mathrm{II}}$ Pablo José Francisco Pena Rodrigues ${ }^{\mathrm{III}}$, João Marcelo Alvarenga Braga ${ }^{\mathrm{III}}$}

\begin{abstract}
Resumo
Os núcleos de vegetação são estruturas formadas espontaneamente por pequenos agrupamentos de indivíduos arbustivos e arbóreos, que, ao evoluírem em pastagens perturbadas, podem contribuir na sucessão ecológica. Para compreender a evolução dos processos de restauração florestal passiva em ecossistemas tropicais, depois de paralisada a pecuária extensiva, foram estudados ambientes com baixa oferta de atributos ambientais localizados na vertente norte dos morrotes isolados entre planície de inundação. Após 40 anos de abandono, os núcleos de vegetação colonizaram $20 \%$ dos ecossistemas graminoides amostrados, apresentando-se em estágio moderado de perturbação. A composição florística dos estratos superior e regenerante evidenciou que núcleos com duas ou mais espécies florestais pioneiras e com síndrome de dispersão anemocórica, como Moquiniastrum polymorphum, apresentam alta probabilidade de terem seus processos de restauração catalisados no tempo. Núcleos desenvolvidos apresentam riqueza e abundância maiores em seu estrato regenerante, além de espécies zoocóricas e não pioneiras, sugerindo interações positivas entre processos ecológicos, condição que confere sustentabilidade aos avanços da restauração florestal passiva. O aprimoramento destas informações pode contribuir para o desenvolvimento de modelos de restauração induzidos com base nos efeitos da nucleação.
\end{abstract}

Palavras-chave: Regeneração natural; Sucessão ecológica; Nucleação

\begin{abstract}
The nuclei of vegetation are structures spontaneously formed by small clusters of individual shrubs and trees that, upon evolving in disturbed pastures can contribute to the ecological succession. In order to understand the evolution of passive restoration processes in tropical ecosystems, after extensive cattle ranching was stopped, environments with low environmental attributes were studied, located in the northern slope of the isolated mounds between floodplain. After 40 years of abandonment, the nuclei of vegetation colonized $20 \%$ of the graminoid ecosystems sampled, presenting at a moderate stage of disturbance. The floristic composition of the upper strata and regenerating showed that nuclei with two or more pioneer forest species and anemocoric dispersion syndrome, such as Moquiniastrum polymorphum, present a high probability of having their restoration processes catalyzed in time. Developed nuclei present higher richness and abundance in their regenerating stratum, besides zoological and nonpioneering species, suggesting positive interactions between the ecological processes, a condition that gives sustainability to the advances of the passive forest restoration. The improvement of this information can contribute to the development of models of induced restoration based on the effects of nucleation.
\end{abstract}

Keywords: Natural regeneration; Ecological succession; Nucleation

\footnotetext{
Engenheira Florestal, MSc., Doutoranda do Programa de Pós-Graduação em Ciências Ambientais e Florestais, Instituto de Florestas, Universidade Federal Rural do Rio de Janeiro, BR 465, Km 07, CEP 23890-000 Seropédica (RJ), Brasil. pollyros@gmail.com (ORCID: 0000-0002-7935-3639)

Engenheiro Florestal, Dr., Professor do Departamento de Ciências Ambientais, Instituto de Florestas, Universidade Federal Rural do Rio de Janeiro, BR 465, Km 07, CEP 23890-000, Seropédica (RJ), Brasil. ricval@ufrrj.br (ORCID: 0000-0002-7209-8815)

III Biólogo, Dr., Pesquisador do Instituto de Pesquisas Jardim Botânico do Rio de Janeiro, Rua Pacheco Leão, 915, CEP 22460-030, Rio de Janeiro (RJ), Brasil. pablojfpr@hotmail.com (ORCID: 0000-0001-8054-3643) / jmabraga@jbrj.gov.br (ORCID: 0000-0002-6124-1970)
} 


\section{Introdução}

O aumento populacional intensificou a pressão de uso dos recursos do bioma Mata Atlântica, principalmente com a substituição das florestas para estabelecimento de culturas agrícolas, pecuária e expansão urbana, o que afetou diretamente a vegetação e suas funcionalidades (GROENEVELD et al., 2009; LIRA et al., 2012; SCARANO; CEOTTO, 2015). Por conta disso, sua configuração é composta predominantemente por fragmentos florestais inferiores a 50 ha (RIBEIRO et al., 2009), interpostos por áreas em processo de degradação, tendendo a se tornar ecossistemas perturbados (CORTINES; VALCARCEL, 2009; MIRANDA et al., 2011). Isto se torna um agravante por interromper a conectividade entre fragmentos e funções ecossistêmicas do seu habitat original (ARONSON; DURIGAN; BRANCALION, 2011).

A restauração ecológica, definida como o processo que auxilia o restabelecimento de ecossistemas degradados, danificados ou destruídos (SOCIETY FOR ECOLOGICAL RESTORATION INTERNATIONAL, 2004), se torna uma ferramenta de recuperação. A restauração ativa se caracteriza pela introdução de diásporos e plantio direto, acelerando o processo de restauração (DESIMONE, 2011). Entretanto, os custos de implementação e manutenção podem ser fatores limitantes (HOLL; AIDE, 2011).

A restauração passiva é definida como o retorno espontâneo de um ecossistema perturbado a um estado preexistente, utilizando a interrupção do agente de perturbação e manejo da resiliência remanescente, cuja forma e funções se ajustam em equilíbrio dinâmico que convergem para restituição da estrutura anterior à perturbação (WESTMAN, 1978; CORTINES; VALCARCEL, 2009; ARONSON; DURIGAN; BRANCALION, 2011; SCOWCROFT; YEH, 2013). Como a restauração passiva ocorre em áreas em estado de abandono, a remoção de gado e interrupção de práticas da agricultura são formas de intervenção capazes de alavancar o estabelecimento da sucessão secundária (SHONO; CADAWENG; DURST, 2007; MORRISON; LINDELL, 2010; BAYLÃO JÚNIOR; VALCARCEL; NETTESHEIN, 2013), promovendo a aquisição de resiliência para estimular o autodesenvolvimento dos ecossistemas.

A relação entre competição e facilitação constitui uma das mais importantes interações para o estabelecimento da sucessão (CONNELL; SLATYER, 1977). Plantas que crescem próximas umas das outras e que competem entre si, geram efeitos negativos que limitam o acesso à água, nutrientes, luz e espaço (PADILLA; PUGNAIRE, 2006). A principal competição nas áreas de pastagem ocorre pela dominância de gramíneas invasoras, afetando a sobrevivência de plântulas de espécies ambientalmente mais exigentes, dificultando a chegada de propágulos, germinação de sementes e o estabelecimento de espécies arbóreas (HOLL, 2002; CHEUNG; MARQUES; LIEBSCH, 2009). Já a facilitação pode exercer influências positivas por modificar as condições bióticas, aumentando a sobrevivência de indivíduos e a diversidade local (STACHOWICZ, 2001; PADILLA; PUGNAIRE, 2006; BAYLÃO JÚNIOR; VALCARCEL; NETTESHEIN, 2013; MCINTIRE; FAJARDO, 2014). Desta forma, as espécies facilitadoras colonizam uma área em estágio inicial e melhoram o ambiente para o seu desenvolvimento, contribuindo para a evolução dos ecossistemas (BAYLÃO JÚNIOR; VALCARCEL; NETTESHEIN, 2013).

Indivíduos arbóreos remanescentes em pastagens abandonadas podem facilitar o restabelecimento da restauração em núcleos (REIS et al., 2003), tanto pela chegada, como pelo estabelecimento e desenvolvimento de espécies (YARRANTON; MORRISON, 1974; BAYLÃO JÚNIOR; VALCARCEL; NETTESHEIN, 2013). Entretanto, o desenvolvimento de grupos de indivíduos arbustivos e arbóreos em pastagens depende do estabelecimento do primeiro indivíduo e de seus atributos, que irão condicionar o desenvolvimento dos demais. Eles podem reduzir a competição interespecífica entre gramíneas e estimular a regeneração natural, geram sombra que reduz os efeitos das oscilações térmicas diárias, contribuem com a oferta de matéria orgânica e ciclagem de nutrientes, além de oferecer abrigo e alimento para animais dispersores de sementes e atrair polinizadores (KOLB, 1993; CALLAWAY, 1995; REIS et al., 2003; BAYLÃO JÚNIOR; VALCARCEL; NETTESHEIN, 2013; FIGUEIREDO et al., 2014). 
Entender os processos ecológicos em áreas perturbadas tropicais permite aperfeiçoar técnicas de restauração florestal sustentáveis, baseado nas teorias de sucessão (HOLL, 2002). Um dos principais desafios é harmonizar o grupo de espécies com potencial para a expansão florestal e suas interações positivas para áreas adjacentes, reduzindo os efeitos da fragmentação e aumentando a biodiversidade (BAYLÃO JÚNIOR; VALCARCEL; NETTESHEIN, 2013; SCOWCROFT; YEH, 2013; FIGUEIREDO et al., 2014; MCINTIRE; FAJARDO, 2014).

Neste contexto, a hipótese deste estudo estabelece que quanto maior for o núcleo de vegetação arbustivo/arbóreo, maior será a abundância e riqueza de espécies, principalmente zoocóricas e não pioneiras. Com isso, o objetivo do presente estudo foi caracterizar a sucessão ecológica envolvendo núcleos de espécies arbustivas e arbóreas, como estratégia de restauração passiva em pastagens abandonadas na Mata Atlântica.

\section{Métodos}

\section{Área de estudo e sítio amostral}

O local estudado foi a Reserva Biológica de Poço das Antas (Rebio Poços das Antas), situada na região de baixada litorânea no município de Silva Jardim, estado do Rio de Janeiro, Brasil (22 $30^{\circ}$ e $22^{\circ} 33^{\prime}$ ' $; 2^{\circ} 15^{\prime}$ e $42^{\circ} 19^{\prime} \mathrm{W}$ ) (Figura 1), clima “As”, segundo Köppen, tropical chuvoso com estação seca no inverno (ARAÚJO et al., 2006) e domínio ecológico da Mata Atlântica. A floresta foi substituída por lavoura de cana-de-açúcar, seguida da pecuária extensiva entre outras culturas. A agricultura foi abandonada pela baixa produtividade, dificuldades de mecanização e vocação regional. O rebaixamento do lençol freático promovido pela retificação da drenagem principal viabilizou parcialmente a agricultura em meados do século XIX, gerando crescimento efêmero da citricultura e pecuária (CUNHA; FREITAS, 2004).

A combinação dos efeitos antrópicos alterou o funcionamento dos ecossistemas de forma direta e indireta, principalmente nos morrotes, por terem solos secos, baixa fertilidade e alto risco de processos erosivos, inviabilizando economicamente seus usos. A consequência foi o aumento da pecuária extensiva e abandono das áreas, que entraram em processo de restauração passiva formando fragmentos florestais de baixa diversidade em áreas que ainda resguardavam mínimas condições ambientais para o estabelecimento da vegetação, sendo assim locais resilientes.

$\mathrm{Na}$ Rebio, estes processos foram potencializados há 40 anos pelos impedimentos legais interpostos pela decretação da Unidade de Conservação, encerrando a exploração e dando início aos processos de restauração passiva. Em áreas menos resilientes predominam gramíneas em competição interespecífica, formando pastagens abandonadas em várzeas intermitentes e/ou saturadas, em que predominam Neossolos flúvicos. Já nos morrotes (altitude inferior a $200 \mathrm{~m}$ ), predominam pastagens compostas principalmente de Melinis minutiflora (capim-gordura) nos Argissolos (MORAES et al., 2006).

Foram selecionados cinco morrotes com pastagens abandonadas e núcleos de vegetação arbustivos e/ou arbóreos de ambientes secos, circunscritos entre áreas úmidas típicas de planície de inundação (Figura 1), dificultando assim a conectividade entre espécies dos fragmentos nos diferentes ambientes. Nestes morrotes, amostrou-se a face norte, cujo estresse hídrico em decorrência da maior intensidade de radiação solar constitui uma importante limitação de atributos ecológicos, que afeta o desenvolvimento da vegetação. Nestas áreas de meia encosta $(60 \mathrm{~m}<$ altitude $<120 \mathrm{~m})$ foram alocadas seis parcelas permanentes $(60 \mathrm{~m} \times 30 \mathrm{~m})$, nas quais se realizou censo dos indivíduos arbustivos e arbóreos em 1,08 ha de área amostrada, representando $0,02 \%$ da área similar na região. 


\section{Figura 1 - Localização das áreas de coleta em morrotes situados em baixada litorânea na Reserva Biológica de Poço das Antas, estado do Rio de Janeiro, Brasil.}

Figure 1 - Location of collection areas on mounds situated in the coastal plain at Poço das Antas Biological Reserve, state of Rio de Janeiro, Brazil.

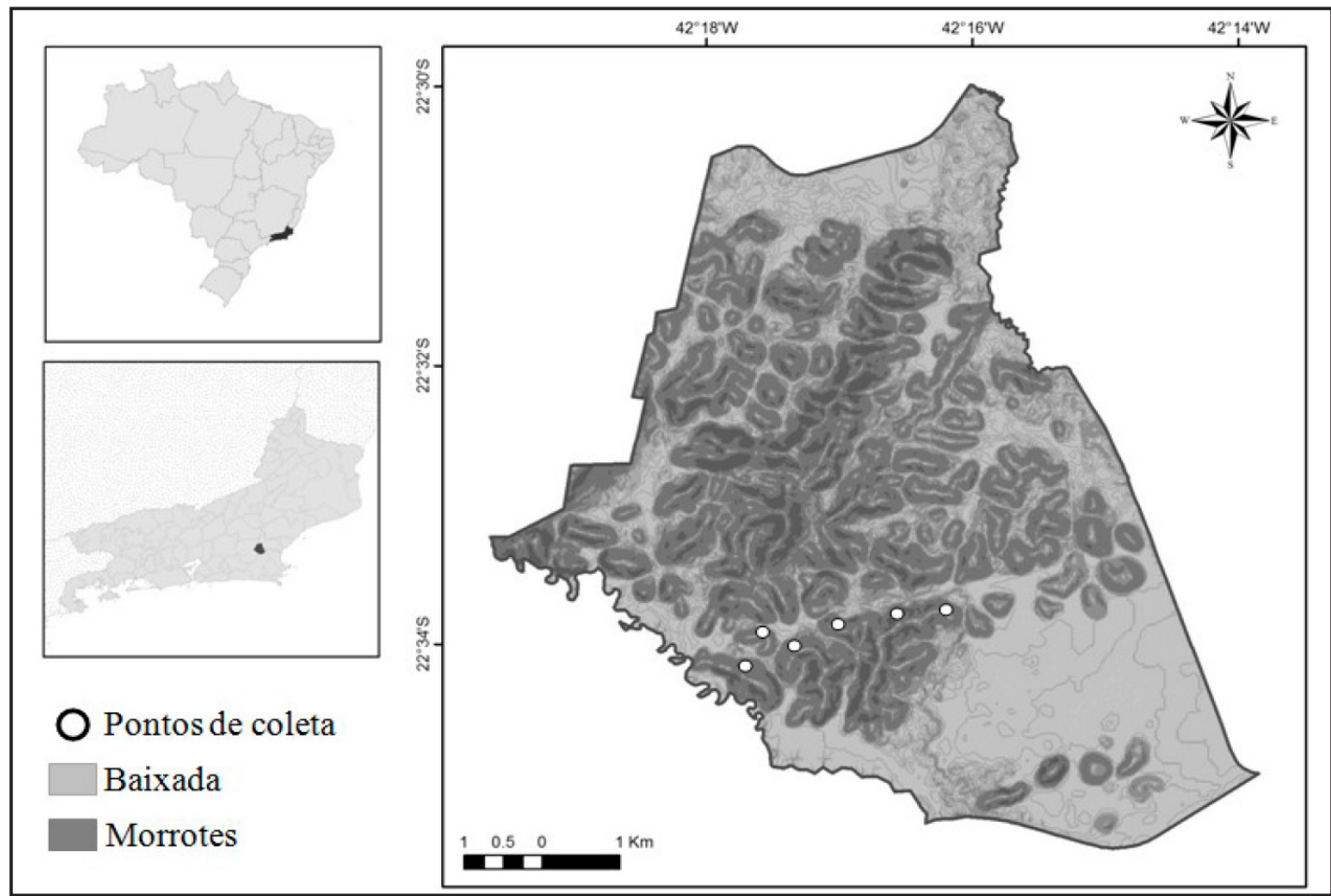

Fonte: Autores (2019)

\section{Núcleos de vegetação}

Foi considerado núcleo de vegetação arbustivo/arbóreo os agrupamentos com no mínimo dois indivíduos arbustivos ou arbóreos que apresentavam sobreposição de copas e/ou com 0,50 metros de afastamento. Foi medida a altura de cada núcleo de vegetação e a quantidade de indivíduos que formavam os núcleos e quantos se encontravam de forma isolada na pastagem. Foi medido também o maior e menor diâmetro ( $\mathrm{Da}$ e $\mathrm{Db})$, obtendo a área a partir da elipse $\left(\pi^{\star} \mathrm{Da} / 2^{\star} \mathrm{Db} / 2\right)$, conforme literatura (KOLB, 1993; MAZA-VILLALOBOS; BALVANERA; MARTÍNEZ-RAMOS, 2011). Os núcleos foram classificados em função do tamanho da projeção de suas copas em pequeno $\left(<23 \mathrm{~m}^{2}\right)$, médio $\left(23-50 \mathrm{~m}^{2}\right)$ e grande $\left(>50 \mathrm{~m}^{2}\right)$, e foram georreferenciados e projetados sobre base cartográfica planialtimétrica.

\section{Composição do estrato superior e regenerante}

Os indivíduos adultos de porte arbustivo e arbóreo com diâmetro a altura do peito (DAP) superior a $2,5 \mathrm{~cm}$ e altura mínima de 1,5 metros foram considerados estrato superior, dispostos tanto de forma isolada como em núcleos.

Os indivíduos arbustivos e arbóreos juvenis e do banco de plântulas, com tamanho entre 0,10 e 1,5 metros de altura, e diâmetro a altura do solo (DAS) inferior a 2,5 cm, foram considerados estrato regenerante.

Os indivíduos dos dois estratos foram identificados, utilizando o sistema de classificação 
baseado no APG III (ANGIOSPERM PHYLOGENY GROUP, 2009), a partir de observações no campo, literaturas, consultas a coleções e auxílio de especialistas. As espécies foram classificadas quanto ao grupo sucessional em pioneiras e não pioneiras (SWAINE; WHITMORE, 1988) e síndrome de dispersão, a partir de revisão bibliográfica e característica dos frutos.

Para o estrato superior foi realizada a fitossociologia das espécies, calculando-se o valor de importância (VI). A avaliação estrutural foi feita segundo Moro e Martins (2011).

\section{Análise dos dados}

As relações e influências presentes no processo de sucessão em pastagens abandonadas foram verificadas pelos testes de correlação de Pearson (r) para os parâmetros: área do núcleo de vegetação e abundância do estrato superior, área do núcleo de vegetação e riqueza do estrato superior, riqueza do estrato superior e riqueza do estrato regenerante, área do núcleo de vegetação e abundância do estrato regenerante, abundância do estrato superior e abundância do estrato regenerante.

O teste "análise de espécies indicadoras" foi feito de forma pareada entre núcleos, com seleção aleatória por sorteio dos núcleos escolhidos. As análises estatísticas foram feitas no software R 2.13.2 (R DEVELOPMENT CORE TEAM, 2013), utilizando-se os pacotes labdsv (ROBERTS, 2013) para realização do teste de análise de espécies indicadoras e vegan 2.0-9 (OKSANEN et al., 2013) para os demais testes.

\section{Resultados e discussão}

Foram encontrados 50 núcleos de vegetação nas meias encostas dos morrotes situados na exposição de face norte, em áreas em que não houve reflorestamento, totalizando 2.121,07 $\mathrm{m}^{2}$ de restauração passiva (cerca de $20 \%$ da área amostrada). Os $80 \%$ remanescentes apresentam pastagens com predominância de Melinis minutiflora (capim-gordura), em diferentes graus de perturbação, mesmo tendo cessada a pecuária há 40 anos.

Foram observados 16 núcleos pequenos, 17 médios e 17 grandes. Dois núcleos, um pequeno e um grande, por possuírem características discrepantes dos demais (referente à composição e tamanho excessivo), não foram utilizados nas análises e discussões. Foram encontrados 468 indivíduos compondo o estrato superior, divididos em 23 famílias e 33 espécies. Desses indivíduos, 168 se encontravam isolados entre matriz graminoide e 300 formavam núcleos de vegetação.

$\mathrm{O}$ aumento em área dos núcleos evidenciou maior riqueza de espécies (Figura 2), que causa maior sustentabilidade do processo de restauração. Cheung, Liebsch e Marques (2010) encontraram resultado semelhante, em que a sucessão em pastagem abandonada foi caracterizada pelo aumento do número de árvores de espécies zoocóricas e não pioneiras.

A abundância da espécie Moquiniastrum polymorphum associada ao domínio de espécies pioneiras $(70 \%)$ nas comunidades evidencia que os núcleos se encontram em estágio inicial de sucessão (Tabela 1). Já a prevalência da síndrome de dispersão zoocórica em $75 \%$ das espécies, sinaliza presença de fauna dispersora entre núcleos e remanescentes de fragmentos florestais de áreas resilientes.

Ter encontrado uma única espécie que ocorreu somente de forma isolada (Myrcia sp.), representada por um indivíduo, infere que independentemente da espécie, a tendência de um indivíduo isolado é formar núcleos de vegetação. Isso pode ser explicado pelos indivíduos isolados trazerem benefícios às áreas colonizadas (GARCÍA-ORTH; MARTÍNEZ-RAMOS, 2011; DERROIRE et al., 2016), o que pode gerar processos de facilitação que favorecem a formação dos núcleos. A espécie Moquiniastrum polymorphum apresentou maior VI e conjuntamente com Psidium guineense e Albizia polycephala explicam $72,4 \%$ do VI das espécies isoladas, bem como com Psidium guajava e Psidium guineense explicam 50,1\% do VI dos núcleos (Tabela 2). 


\section{Figura 2 - Evolução sucessional em pastagem abandonada.}

Figure 2 - Successional evolution in abandoned pasture.

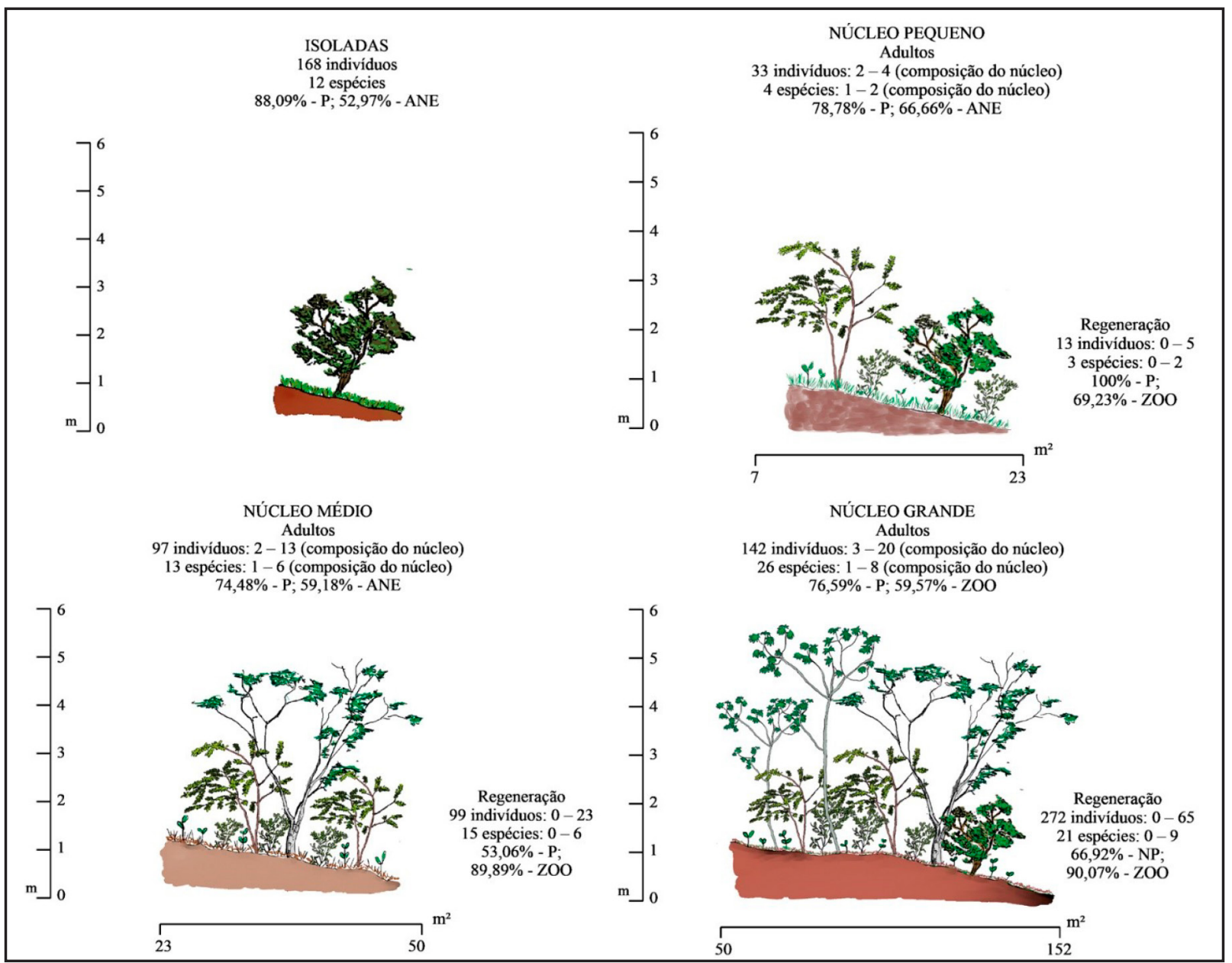

Fonte: Autores (2019)

Legenda: grupo sucessional: P - pioneira, NP - não pioneira; síndrome de dispersão: ANE - anemocórica, ZOO zoocórica.

Legend: successional group: P - pioneer, NP - non- pioneer; dispersion syndrome: ANE - anemocoric, ZOO - zoocoric.

Tabela 1 - Composição de espécies arbustivo/arbóreas presentes no estrato superior em pastagem abandonada.

Table 1 - Composition of shrub/tree species present in the upper stratum in abandoned pasture.

\begin{tabular}{|c|c|c|c|c|}
\hline FAMÍLIA/ESPÉCIE & ABUND & GS & SD & FO \\
\hline ANACARDIACEAE & & & & \\
\hline Schinus terebinthifolius Raddi & 9 & $P$ & $\mathrm{ZOO}$ & $\mathrm{NV}$ \\
\hline Tapirira guianensis Aubl. & 1 & $\mathrm{P}$ & ZOO & $\mathrm{NV}$ \\
\hline ANNONACEAE & & & & \\
\hline Xylopia sericea A.St.-Hil. & 2 & $\mathrm{P}$ & $\mathrm{ZOO}$ & NV \\
\hline $\begin{array}{l}\text { APOCYNACEAE } \\
\text { Tabernaemontana laeta Mart. }\end{array}$ & 6 & $P$ & $\mathrm{ZOO}$ & $\mathrm{I}, \mathrm{NV}$ \\
\hline
\end{tabular}


Tabela 1 - Continua...

Table 1 - Continued...

\begin{tabular}{|c|c|c|c|c|}
\hline FAMÍLIA/ESPÉCIE & ABUND & GS & SD & FO \\
\hline $\begin{array}{l}\text { ARECACEAE } \\
\text { Attalea humilis Mart. }\end{array}$ & 8 & $\mathrm{P}$ & $\mathrm{ZOO}$ & I, NV \\
\hline $\begin{array}{l}\text { ASTERACEAE } \\
\text { Baccharis dracunculifolia DC. } \\
\text { Moquiniastrum polymorphum (Less.) G. Sancho }\end{array}$ & $\begin{array}{c}23 \\
205\end{array}$ & $\begin{array}{l}P \\
P\end{array}$ & $\begin{array}{l}\text { ANE } \\
\text { ANE }\end{array}$ & $\begin{array}{l}\text { I, NV } \\
\text { I, NV }\end{array}$ \\
\hline $\begin{array}{l}\text { BIGNONIACEAE } \\
\text { Cybistax antisyphilitica (Mart.) Mart. } \\
\text { Handroanthus chrysotrichus (Mart. ex DC.) Mattos } \\
\text { Sparattosperma leucanthum (Vell.) K.Schum. }\end{array}$ & $\begin{array}{l}2 \\
2 \\
2\end{array}$ & $\begin{array}{c}\mathrm{P} \\
\mathrm{NP} \\
\mathrm{P}\end{array}$ & $\begin{array}{l}\text { ANE } \\
\text { ANE } \\
\text { ANE }\end{array}$ & $\begin{array}{l}\mathrm{I}, \mathrm{NV} \\
\mathrm{NV} \\
\mathrm{I}, \mathrm{NV}\end{array}$ \\
\hline $\begin{array}{l}\text { CANNABACEAE } \\
\text { Trema micrantha }(\text { L. }) \text { Blume }\end{array}$ & 7 & $\mathrm{P}$ & ZOO & $\mathrm{NV}$ \\
\hline $\begin{array}{l}\text { FABACEAE } \\
\text { Albizia polycephala (Benth.) Killip ex Record } \\
\text { Dalbergia nigra (Vell.) Allemão ex Benth. } \\
\text { Inga edulis Mart. }\end{array}$ & $\begin{array}{c}26 \\
1 \\
2\end{array}$ & $\begin{array}{l}\mathrm{P} \\
\mathrm{NP} \\
\mathrm{P}\end{array}$ & $\begin{array}{l}\text { AUTO } \\
\text { ANE } \\
\text { ZOO }\end{array}$ & $\begin{array}{l}\mathrm{I}, \mathrm{NV} \\
\mathrm{NV} \\
\mathrm{NV}\end{array}$ \\
\hline $\begin{array}{l}\text { LAURACEAE } \\
\text { Cinnamomum triplinerve (Ruiz \& Pav.) Kosterm. } \\
\text { Nectandra oppositifolia Nees }\end{array}$ & $\begin{array}{c}2 \\
13\end{array}$ & $\begin{array}{l}\mathrm{NP} \\
\mathrm{NP}\end{array}$ & $\begin{array}{l}\mathrm{ZOO} \\
\mathrm{ZOO}\end{array}$ & $\begin{array}{l}\mathrm{NV} \\
\mathrm{I}, \mathrm{NV}\end{array}$ \\
\hline $\begin{array}{l}\text { MELASTOMATACEAE } \\
\text { Miconia albicans }(\text { Sw.) Triana } \\
\text { Miconia cinnamomifolia }(\text { DC.) Naudin }\end{array}$ & $\begin{array}{l}6 \\
7\end{array}$ & $\begin{array}{l}P \\
P\end{array}$ & $\begin{array}{l}\mathrm{ZOO} \\
\mathrm{ZOO}\end{array}$ & $\begin{array}{l}\mathrm{NV} \\
\mathrm{NV}\end{array}$ \\
\hline $\begin{array}{l}\text { MELIACEAE } \\
\text { Guarea guidonia }(\mathbf{L} .) \text { Sleumer }\end{array}$ & 8 & NP & $\mathrm{ZOO}$ & $\mathrm{NV}$ \\
\hline $\begin{array}{l}\text { MYRTACEAE } \\
\text { Myrcia sp. } \\
\text { Myrcia splendens }(\mathrm{Sw} .) \text { DC. } \\
\text { Psidium guajava } \mathrm{L} .{ }^{*} \\
\text { Psidium guineense } \mathrm{Sw} .\end{array}$ & $\begin{array}{c}1 \\
2 \\
48 \\
49\end{array}$ & $\begin{array}{c}- \\
P \\
N P \\
P\end{array}$ & $\begin{array}{l}\mathrm{ZOO} \\
\mathrm{ZOO} \\
\mathrm{ZOO} \\
\mathrm{ZOO}\end{array}$ & $\begin{array}{l}\mathrm{I} \\
\mathrm{NV} \\
\mathrm{I}, \mathrm{NV} \\
\mathrm{I}, \mathrm{NV}\end{array}$ \\
\hline $\begin{array}{l}\text { PERACEAE } \\
\text { Pera glabrata (Schott) Poepp. ex Baill. }\end{array}$ & 7 & $\mathrm{P}$ & ZOO & $\mathrm{I}, \mathrm{NV}$ \\
\hline $\begin{array}{l}\text { PRIMULACEAE } \\
\text { Myrsine coriacea }(\text { Sw.) R.Br. ex Roem. \& Schult. }\end{array}$ & 4 & $\mathrm{P}$ & ZOO & $\mathrm{NV}$ \\
\hline $\begin{array}{l}\text { RUBIACEAE } \\
\text { Tocoyena bullata (Vell.) Mart. }\end{array}$ & 1 & NP & ZOO & $\mathrm{NV}$ \\
\hline $\begin{array}{l}\text { RUTACEAE } \\
\text { Zanthoxylum rhoifolium Lam. }\end{array}$ & 1 & $\mathrm{P}$ & $\mathrm{ZOO}$ & $\mathrm{NV}$ \\
\hline $\begin{array}{l}\text { SALICACEAE } \\
\text { Casearia sylvestris Sw. }\end{array}$ & 4 & $P$ & $\mathrm{ZOO}$ & $\mathrm{NV}$ \\
\hline $\begin{array}{l}\text { SAPINDACEAE } \\
\text { Cupania racemosa (Vell.) Radlk. }\end{array}$ & 3 & $\mathrm{P}$ & $\mathrm{ZOO}$ & $\mathrm{NV}$ \\
\hline $\begin{array}{l}\text { SIPARUNACEAE } \\
\text { Siparuna guianensis Aubl. }\end{array}$ & 9 & NP & $\mathrm{ZOO}$ & $\mathrm{NV}$ \\
\hline
\end{tabular}


Tabela 1 - Conclusão...

Table 1 - Conclusion...

\begin{tabular}{lcccc}
\hline \multicolumn{1}{c}{ FAMÍLIA/ESPÉCIE } & ABUND & GS & SD & FO \\
\hline $\begin{array}{l}\text { SOLANACEAE } \\
\text { Solanum sp. }\end{array}$ & 1 & - & ZOO & NV \\
$\begin{array}{l}\text { STYRACACEAE } \\
\text { Styrax glabratum Schott }\end{array}$ & 2 & - & ZOO & NV \\
$\begin{array}{l}\text { URTICACEAE } \\
\text { Cecropia pachystachya } \text { Trécul }\end{array}$ & 4 & P & ZOO & I, NV \\
\hline
\end{tabular}

Legenda: abundâncias (ABUND); grupo sucessional (GS): pioneira (P), não pioneira (NP); síndrome de dispersão (SD): zoocórica (ZOO), anemocórica (ANE), autocórica (AUTO); forma de ocorrência (FO): isoladas (I), núcleos de vegetação $(\mathrm{NV})$; *espécie exótica.

Legend: abundance (ABUND); successional group (GS): pioneer (P), non-pioneer (NP); dispersal syndrome (SD): zoocoric (ZOO), anemocoric (ANE), autocoric (AUTO); form of occurrence (FO): isolated (I), nuclei of vegetation (NV);

* exotic species.

\section{Tabela 2 - Parâmetros fitossociológicos das cinco espécies representativas amostradas isoladas e formando o estrato superior de núcleos de vegetação arbustivo/arbóreo em pastagens abandonadas.}

Table 2 - Phytosociological parameters of the five representative isolated species and forming the upper stratum of shrub/arboreal nuclei of vegetation in abandoned pastures.

\begin{tabular}{|c|c|c|c|c|c|c|c|c|}
\hline & & Espécies & $\mathrm{Ni}$ & $\mathbf{A b}$ & DR & FR & DoR & VI \\
\hline \multirow{5}{*}{ 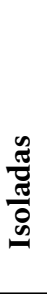 } & 1 & Moquiniastrum polymorphum & 72 & 0,3155 & 43,11 & 27,27 & 51,37 & 40,59 \\
\hline & 2 & Psidium guineense & 35 & 0,0342 & 20,96 & 22,73 & 5,57 & 16,42 \\
\hline & 3 & Albizia polycephala & 17 & 0,1930 & 10,18 & 4,55 & 31,42 & 15,38 \\
\hline & 4 & Baccharis dracunculifolia & 15 & 0,0204 & 8,98 & 9,09 & 3,33 & 7,13 \\
\hline & 5 & Psidium guajava & 17 & 0,0225 & 10,18 & 4,55 & 3,66 & 6,13 \\
\hline \multirow{5}{*}{$\frac{\mathscr{d}}{\frac{d}{0}}$} & 1 & Moquiniastrum polymorphum & 133 & 1,2646 & 45,39 & 9,09 & 61,27 & 38,59 \\
\hline & 2 & Psidium guajava & 31 & 0,0732 & 10,58 & 5,45 & 3,55 & 6,53 \\
\hline & 3 & Psidium guineense & 14 & 0,0192 & 4,78 & 9,09 & 0,93 & 4,93 \\
\hline & 4 & Nectandra opositifolia & 11 & 0,0915 & 3,75 & 5,45 & 4,43 & 4,55 \\
\hline & 5 & Albizia polycephala & 9 & 0,1687 & 3,07 & 1,82 & 8,17 & 4,35 \\
\hline
\end{tabular}

Legenda: $\mathrm{Ni}$ - número de indivíduos; $\mathrm{Ab}$ - área basal $\left(\mathrm{m}^{2}\right)$; DR - densidade relativa (\%); FR - frequência relativa $(\%)$; DoR - dominância relativa (\%); VI - valor de importância (\%).

Legend: $\mathrm{Ni}$ - number of individuals; $\mathrm{Ab}$ - basal area $\left(\mathrm{m}^{2}\right)$; DR - relative density (\%); FR - relative frequency (\%); DoR relative dominance (\%); VI - value of importance (\%).

O elevado número de indivíduos de Moquiniastrum polymorphum presente de forma isolada e em grupos sugere a importância da espécie na formação dos núcleos. Por se tratar de uma espécie pioneira, presente em local com elevada abundância de gramíneas, indica habilidade competitiva para colonizar e se estabelecer, e com isto, melhorar as condições ambientais locais. Como as pastagens voltadas para a vertente norte são ambientes secos, outra propriedade importante da espécie é sua elevada resistência ao fogo (SÁNCHEZ-TAPIA, 2011). Esta espécie também atua quando encontrada nas bordas das capoeiras, 
gerando condições para o desenvolvimento de indivíduos de estágios sucessionais avançados (NEVES; PEIXOTO, 2008).

A interação entre Moquiniastrum polymorphum com outras espécies, como Psidium guajava, estimula os mecanismos de facilitação e permite o estabelecimento diversificado de plântulas, sustentando a manutenção dos núcleos em processo de restauração passiva. Segundo Zahawi e Augspurger (1999), a riqueza de espécies, assim como o número de indivíduos de plântulas, foi consideravelmente maior em pastos compostos por cobertura de Psidium guajava do que em pastos abertos, sugerindo comportamento facilitador da espécie. Outras espécies, como Clusia hilariana em restinga (GARBIN et al., 2012) e Clidemia urceolata em morrotes em áreas de pastagem (MIRANDA et al., 2011), se diferenciam funcionalmente e ofertam propriedades emergentes capazes de permitir o estabelecimento de novos indivíduos.

Os núcleos mais desenvolvidos apresentam maior riqueza de espécies (Figura 3), evidenciando os efeitos sinérgicos entre espécies em seus processos de facilitação e restauração, sendo que a presença exclusiva de algumas espécies nestes núcleos ratifica esta premissa, mesmo tendo a Moquiniastrum polymorphum como a mais abundante nas quatro situações.

\section{Figura 3 - Curva de diversidade e dominância para indivíduos isolados e núcleos de vegetação arbustivo/arbóreo de diferentes tamanhos.}

Figure 3 - Diversity and dominance curve for isolated individuals and shrub/arboreal nuclei of vegetation of different sizes.

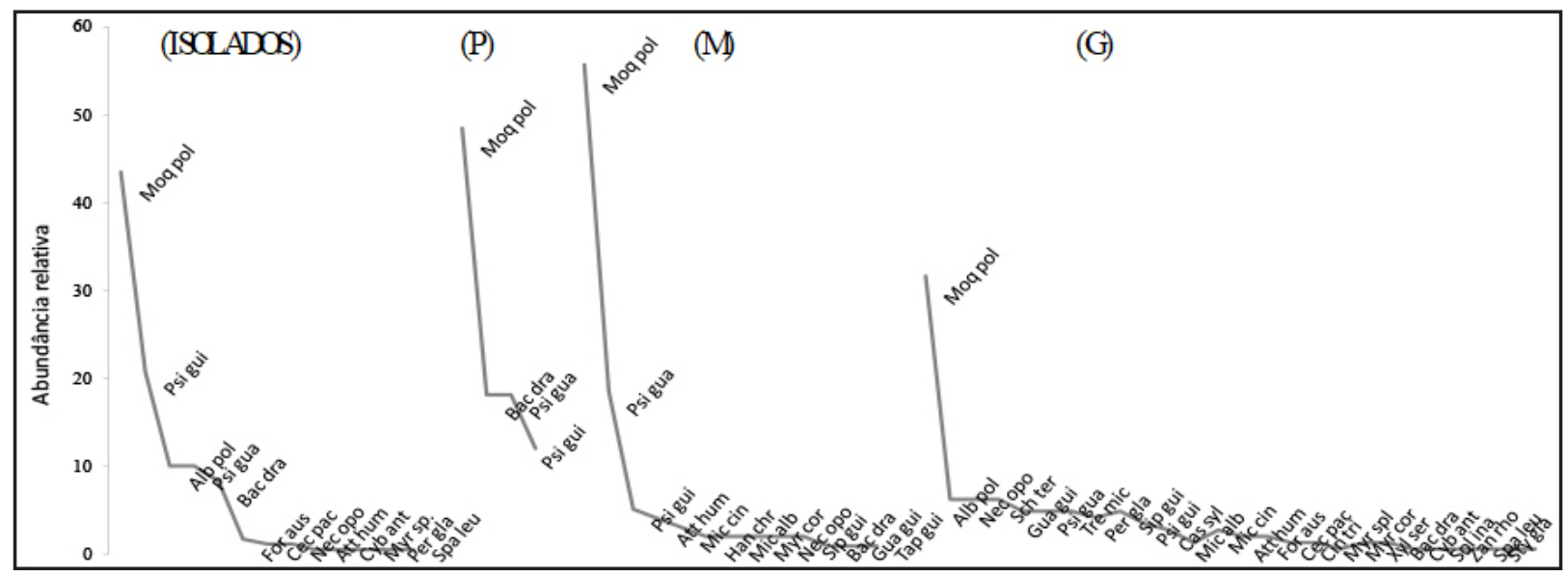

Fonte: Autores (2019)

Legenda: pequeno - P, médio - $M$, grande - G

Legend: small - P, medium - M, large - G

A área do núcleo apresentou melhor correlação com a abundância de árvores ( $\mathrm{r}=0,67$; DF = $46 ; p<0,0001)$ do que com a riqueza do estrato superior $(r=0,49 ; D F=46 ; p=0,00039)$. Em ambas as situações, a correlação foi positiva, evidenciando que quanto maior for a área, maior será a abundância e riqueza. Assim, o desenvolvimento do núcleo ocorre principalmente com o aumento da abundância, mas também de sua riqueza, inferindo que eles tendem a crescer não somente pela dominância das espécies que os compõem, mas também pela chegada de novas espécies vindas de outra fonte de propágulo. Como núcleos maiores atraem dispersores de sementes, estes tendem a apresentar maior probabilidade de se estabelecerem e colonizarem novas áreas (ZAHAWI; AUGSPURGER, 2006). Isto fica claro ao observar a análise de espécies indicadoras (Tabela 3), por meio da qual se verificou em núcleos pequenos uma espécie pioneira, enquanto que as espécies indicadoras de núcleos grandes são espécies não pioneiras. Esta condição indica que a espécie Baccharis dracunculifolia contribui para a formação dos núcleos, enquanto que Siparuna guianensis e Nectandra opositifolia ingressam quando os núcleos já estão estabelecidos e apoiam no seu desenvolvimento. 


\section{Tabela 3 - Análises de espécies indicadoras das diferentes classes de núcleos de vegetação arbustivo/arbóreo.}

Table 3 - Indicator species analysis of the different classes of shrub/arboreal nuclei of vegetation.

\begin{tabular}{lcccccc}
\hline \multicolumn{1}{c}{ Espécie } & $\mathbf{P}(\%)$ & $\mathbf{M}(\%)$ & $\mathbf{G}(\%)$ & Maior \% indicador & $\begin{array}{c}\text { Classe } \\
\text { indicada }\end{array}$ & P valor \\
\hline Siparuna guianensis & 0,000 & 0,000 & 0,333 & 0,333 & $\mathrm{G}$ & 0,004 \\
Baccharis dracunculifolia & 0,259 & 0,007 & 0,007 & 0,259 & $\mathrm{P}$ & 0,052 \\
Nectandra opositifolia & 0,000 & 0,007 & 0,240 & 0,240 & $\mathrm{G}$ & 0,058 \\
\hline
\end{tabular}

Legenda: $\mathrm{P}$ - pequeno, $\mathrm{M}$ - médio, $\mathrm{G}$ - grande.

Legend: P - small, $\mathrm{M}$ - medium, G - large.

A área do núcleo está associada à dinâmica observada nos processos sucessionais (Figura 4), sendo mais intenso nas maiores áreas que nas menores, conforme constatado também por Duarte et al. (2006). Isto é observado nos núcleos grandes, em que a maioria das espécies apresenta dispersão zoocórica (Figura 4b). Isto apresenta elevada importância por impulsionar a chegada de propágulos, principalmente pela fauna dispersora, pois a maior densidade de plantas zoocóricas constitui fator de atração da fauna, e com a chegada dos dispersores há influência na regeneração natural (SANSEVERO et al., 2011). Este aumento também foi constatado em outras áreas perturbadas (ZAHAWI; AUGSPURGER, 2006; DUARTE et al., 2010; BAYLÃO JÚNIOR; VALCARCEL; NETTESHEIN, 2013; FIGUEIREDO et al., 2014).

Figura 4 - Grupo sucessional (A) e síndrome de dispersão (B) do estrato superior apresentado em porcentagem, para os indivíduos isolados e diferentes classes de núcleos.

Figure 4 - Successional group (A) and dispersion syndrome (B) of the upper stratum presented as percentage, for the isolated individuals and different classes of nuclei.

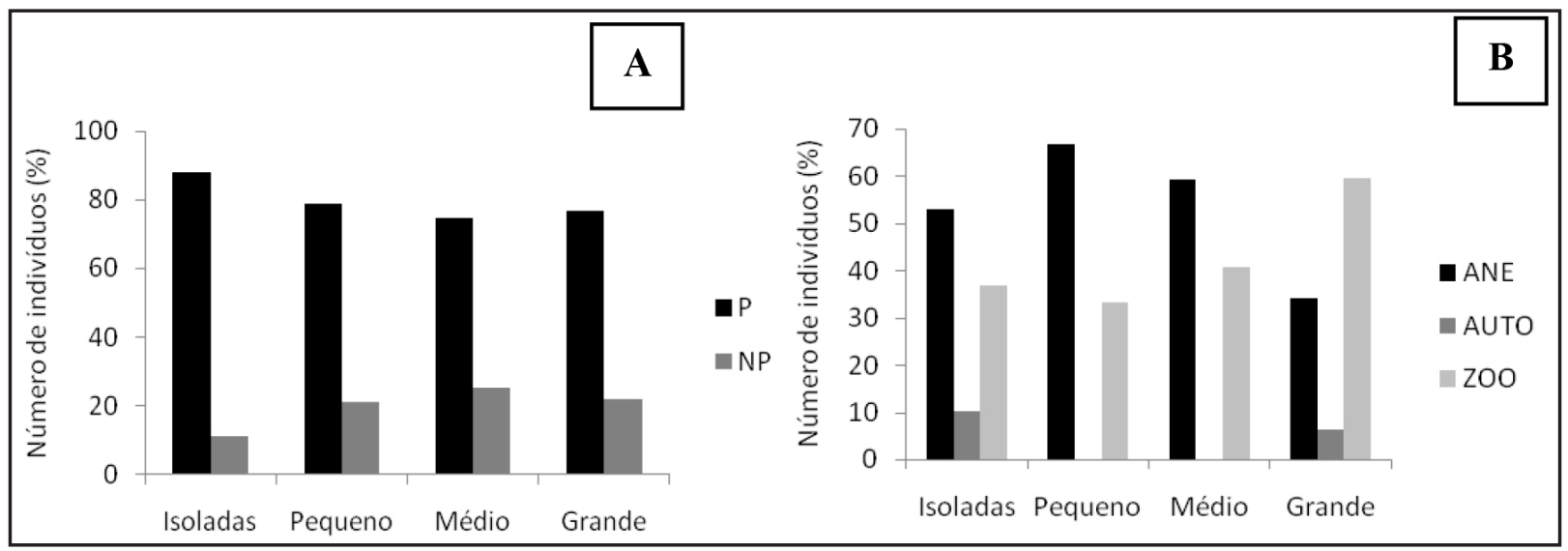

Fonte: Autores (2019)

Legenda: P - pioneiras; NP - não pioneiras; ANE - anemocórica; AUTO - autocórica; ZOO - zoocórica. Legend: P - pioneers; NP - non-pioneer; ANE - anemocoric; AUTO - autocoric; ZOO - zoocoric.

O estrato regenerante apresentou 382 indivíduos, divididos em 14 famílias e 22 espécies vegetais. As espécies que foram encontradas na regeneração são basicamente as mesmas do estrato superior, mudando apenas sua abundância (Tabela 4). 
Tabela 4 - Composição de espécies arbustivo/arbóreas presentes no estrato regenerante dos núcleos de vegetação em pastagem abandonada.

Table 4 - Composition of shrub/tree species present in the regenerating stratum of nuclei of vegetation in abandoned pastures.

\begin{tabular}{|c|c|c|c|c|}
\hline FAMÍLIA/ESPÉCIE & $\mathrm{ABr}$ & ABs & GS & SD \\
\hline \multicolumn{5}{|l|}{ ANACARDIACEAE } \\
\hline Schinus terebinthifolius Raddi & 4 & 9 & $\mathrm{P}$ & $\mathrm{ZOO}$ \\
\hline Tapirira guianensis Aubl. & 2 & 1 & $\mathrm{P}$ & $\mathrm{ZOO}$ \\
\hline \multicolumn{5}{|l|}{ ANNONACEAE } \\
\hline Xylopia sericea A.St.-Hil. & 12 & 2 & $\mathrm{P}$ & $\mathrm{ZOO}$ \\
\hline \multicolumn{5}{|l|}{ ARECACEAE } \\
\hline Attalea humilis Mart. & 1 & 8 & $\mathrm{P}$ & $\mathrm{ZOO}$ \\
\hline \multicolumn{5}{|l|}{ ASTERACEAE } \\
\hline Baccharis dracunculifolia DC. & 2 & 23 & $\mathrm{P}$ & ANE \\
\hline Moquiniastrum polymorphum (Less.) G. Sancho & 18 & 205 & $\mathrm{P}$ & ANE \\
\hline \multicolumn{5}{|l|}{ BIGNONIACEAE } \\
\hline Cybistax antisyphilitica (Mart.) Mart. & 1 & 2 & $\mathrm{P}$ & ANE \\
\hline \multicolumn{5}{|l|}{ EUPHORBIACEAE } \\
\hline Alchornea triplinervia (Spreng) Müll. Arg & 4 & - & $\mathrm{P}$ & $\mathrm{ZOO}$ \\
\hline \multicolumn{5}{|l|}{ FABACEAE } \\
\hline Dalbergia nigra (Vell.) Allemão ex Benth. & 2 & 1 & NP & ANE \\
\hline Albizia polycephala (Benth.) Killip ex Record & 14 & 26 & $\mathrm{P}$ & AUTO \\
\hline \multicolumn{5}{|l|}{ LAURACEAE } \\
\hline Cinnamomum triplinerve (Ruiz \& Pav.) Kosterm. & 63 & 2 & NP & $\mathrm{ZOO}$ \\
\hline Nectandra oppositifolia Nees & 37 & 13 & NP & $\mathrm{ZOO}$ \\
\hline \multicolumn{5}{|l|}{ MELASTOMATACEAE } \\
\hline Clidemia hirta (L.) D.Don & 5 & - & $\mathrm{P}$ & $\mathrm{ZOO}$ \\
\hline Miconia albicans (Sw.) Triana & 22 & 6 & $\mathrm{P}$ & $\mathrm{ZOO}$ \\
\hline Miconia cinnamomifolia (DC.) Naudin & 11 & 7 & $\mathrm{P}$ & $\mathrm{ZOO}$ \\
\hline $\begin{array}{l}\text { MELIACEAE } \\
\text { Guarea guidonia (L.) Sleumer }\end{array}$ & 22 & 8 & NP & $\mathrm{ZOO}$ \\
\hline \multicolumn{5}{|l|}{ MYRTACEAE } \\
\hline Myrcia sp. & 10 & 1 & - & $\mathrm{ZOO}$ \\
\hline Myrcia splendens (Sw.) DC. & 5 & 2 & $\mathrm{P}$ & $\mathrm{ZOO}$ \\
\hline Psidium guajava $\mathbf{L}^{*}$ & 22 & 48 & NP & $\mathrm{ZOO}$ \\
\hline Psidium guineense $\mathrm{Sw}$. & 37 & 49 & $\mathrm{P}$ & $\mathrm{ZOO}$ \\
\hline \multicolumn{5}{|l|}{ PRIMULACEAE } \\
\hline Myrsine coriacea (Sw.) R.Br. ex Roem. \& Schult. & 10 & 4 & $\mathrm{P}$ & $\mathrm{ZOO}$ \\
\hline \multicolumn{5}{|l|}{ SIPARUNACEAE } \\
\hline Siparuna guianensis Aubl. & 78 & 9 & NP & $\mathrm{ZOO}$ \\
\hline
\end{tabular}

Legenda: abundâncias do estrato regenerante $(\mathrm{ABr})$, abundância do estrato superior (ABs); grupo sucessional $(\mathrm{G} S)$ : pioneira $(\mathrm{P})$, não pioneira (NP); síndrome de dispersão $(\mathrm{SD})$ : zoocoria $(\mathrm{ZOO})$, anemocoria (ANE), autocoria (AUTO); *espécie exótica.

Legend: abundances of the regenerating stratum $(\mathrm{ABr})$, abundance of the upper stratum (ABs); successional group (GS): pioneer (P), non-pioneer (NP); dispersal syndrome (SD): zoocoric (ZOO), anemocoric (ANE), autocoric (AUTO); *exotic species. 
Espécies como Cinnamomum triplinerve, Miconia albicans, Guarea guidonia, e Siparuna guianensis se destacaram por terem baixa ocorrência no estrato superior e serem abundantes no estrato regenerante. As espécies Baccharis dracunculifolia, Moquiniastrum polymorphum e Psidium guajava apresentam comportamento antagônico, pela diferença existente em suas abundâncias nos diferentes estratos.

Moquiniastrum polymorphum, que foi a mais abundante entre as arbóreas adultas (205 indivíduos), apresentou apenas 18 indivíduos no estrato regenerante, evidenciando sua amplitude funcional como pioneira no estabelecimento da restauração passiva. A intensa presença de sementes da espécie na chuva de sementes nessa região, condição observada por Kolb (1993), evidencia que o elevado número de propágulos produzido não é suficiente para manter sua dominância no estrato regenerante, até mesmo por conta das novas condições ambientais causadas pelos indivíduos adultos (DERROIRE et al., 2016). Além disso, os indivíduos isolados, assim como árvores remanescentes, se tornam locais de pouso para aves, que trazem sementes de outras espécies de estágios sucessionais mais avançados (GUEVARRA et al., 1986).

O número de indivíduos e espécies da regeneração natural aumenta quanto maior for a área do núcleo (Figura 2), inferindo que os mesmos oferecem subsídios para o estabelecimento de plântulas. Também foi possível observar mudança no padrão da regeneração, com indivíduos pioneiros em núcleos pequenos, enquanto nos núcleos grandes houve maioria de indivíduos não pioneiros (Figura 5a). Entretanto, em todos os núcleos, a maioria dos indivíduos regenerantes apresentou síndrome de dispersão zoocórica (Figura 5b), padrão este diferente do encontrado no estrato superior.

Como anteriormente observado (Figuras 3 e 4), existe um elevado número de indivíduos anemocóricos adultos (Moquiniastrum polymorphum), mas, com o aumento dos núcleos, também há o aumento da presença de espécies zoocóricas. Então, é preciso em um primeiro momento a presença de espécies rústicas e facilitadoras (BAYLÃO JÚNIOR; VALCARCEL; NETTESHEIN, 2013; MCINTIRE; FAJARDO, 2014; BUENO; LLAMBÍ, 2015), geralmente anemocóricas, para ocupar a área, já que as espécies zoocóricas regeneram mais sob indivíduos isolados do que diretamente na pastagem (GUEVARRA et al., 1986; ZAHAWI; AUGSPURGER, 2006). Com o crescimento desses indivíduos compondo os núcleos, a tendência é das espécies zoocóricas na regeneração aumentar, já que o número de regenerantes zoocóricos é maior sob uma árvore de dispersão zoocórica do que sob uma anemocórica (DERROIRE et al., 2016).

\section{Figura 5 - Grupo sucessional (A) e síndrome de dispersão (B) do estrato regenerante apresentado em porcentagem, para as diferentes classes de núcleos.}

Figure 5 - Successional group (A) and dispersion syndrome (B) of the regenerating stratum presented as percentage, for the different classes of nuclei.

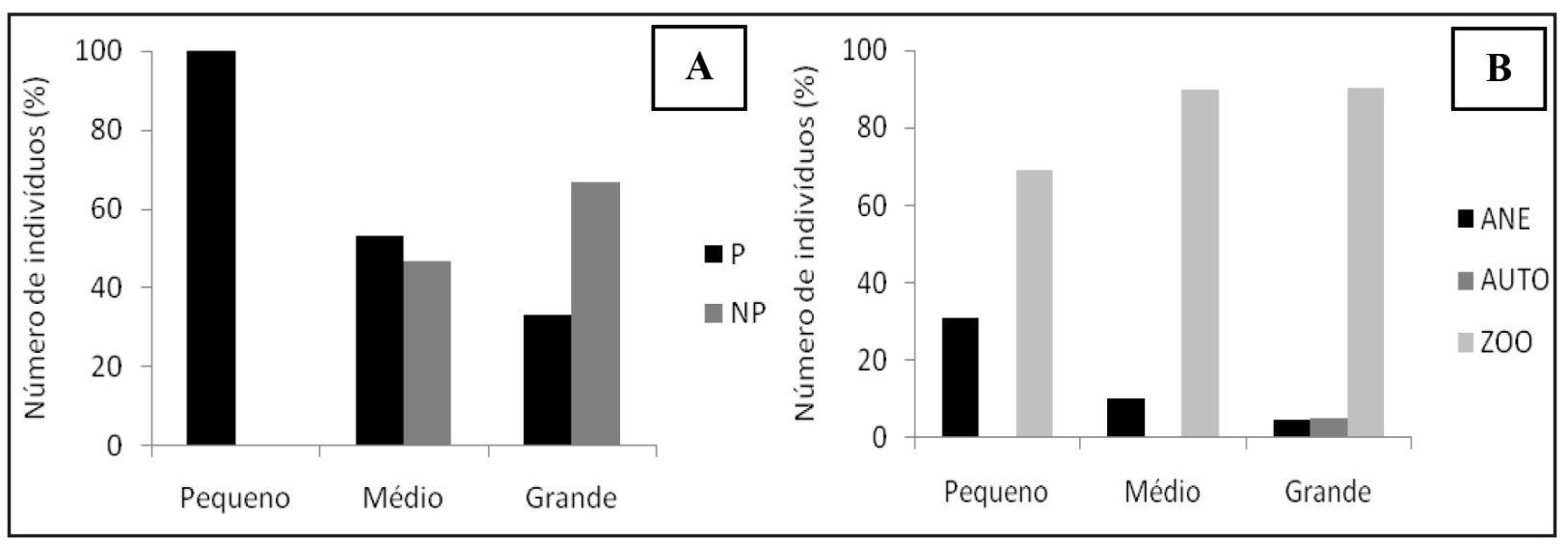

Fonte: Autores (2019)

Legenda: P - pioneiras; NP - não pioneiras; ANE - anemocórica; AUTO - autocórica; ZOO - zoocórica.

Legend: P - pioneers; NP - non-pioneer; ANE - anemocoric; AUTO - autocoric; ZOO - zoocoric. 
Comparando a regeneração natural com o estrato superior, encontrou-se maior correlação entre as abundâncias dos dois estratos $(\mathrm{r}=0,77 ; \mathrm{DF}=46 ; \mathrm{P}<0,0001)$, seguido pela correlação entre as riquezas dos estratos $(\mathrm{r}=0,75 ; \mathrm{DF}=46 ; \mathrm{P}<0,0001)$ e entre a área do núcleo e abundância de regenerantes $(r=0,46 ; \mathrm{DF}=46 ; \mathrm{P}=0,00099)$. Entretanto, somente a correlação entre abundâncias e riqueza de espécies foi significativa.

Para árvores remanescentes, Sandor e Chazdon (2014) não encontraram influência destas sobre a densidade de plântulas ou medidas de crescimento, como área basal. Entretanto, os autores constataram o efeito das árvores remanescentes na composição, diversidade e nos grupos ecológicos da regeneração encontrada em suas proximidades. Mas, ao comparar com áreas abertas, existe um aumento na cobertura vegetal, riqueza e densidade de espécies florestais sob a cobertura de arbustos do que nos espaços existentes entre os mesmos, já que há uma queda na amplitude térmica sob o dossel (BUENO; LLAMBÍ, 2015).

A restauração envolvendo a biologia das espécies e seu comportamento de forma coletiva ocorre a partir da regeneração natural e de suas etapas sucessionais na vegetação, ocasionando mudanças espontâneas na sua composição e estrutura no tempo e espaço (SMITH, 1996; SHONO; CADAWENG; DURST, 2007; SANTOS et al., 2011). Contudo, os núcleos constituem de forma lenta e gradual a restauração florestal passiva de áreas perturbadas e abandonadas, isto porque se trata de uma alternativa que envolve processos de sucessão natural (REIS et al., 2010), e pelas condições adversas encontradas no local, como a competição com gramíneas que influencia na biodiversidade (HOLL et al., 2000; HOLL, 2002; MARTINS; LEITE; HARIDASAN, 2004; BOYD; SVEJCAR, 2011). Mas é a partir dessas áreas que se tem a informação das trajetórias sucessionais das pastagens abandonadas ao longo prazo, já que a regeneração hoje observada será a que vai compor os estágios futuros (DERROIRE et al., 2016).

\section{Conclusões}

Para a área de estudo, composta por pastagens abandonadas presentes em encostas com baixa oferta de atributos ambientais, a dinâmica sucessional em núcleos de vegetação de porte arbustivo/arbóreo evidenciam que, quando há requisitos mínimos de resiliência, existe o potencial para o início e estabelecimento natural da vegetação florestal. A espécie pioneira Moquiniastrum polymorphum tem papel funcional determinante desde o início do estabelecimento natural do núcleo de forma isolada até o seu desenvolvimento, quando cede espaço e funções às espécies de estágios mais evoluídos.

Nesses locais, a nucleação como mecanismo de restauração florestal passiva se mostrou eficiente, embora seu desenvolvimento seja lento, totalizando $20 \%$ de ocupação na matriz graminoide após 40 anos de abandono. Os núcleos de vegetação podem ser utilizados como elemento diagnóstico preliminar em projetos de restauração induzida. Os efeitos da nucleação podem ser catalisados a partir do plantio de espécies florestais compatíveis com seus mecanismos de facilitação e com a dinâmica sucessional de florestas mais evoluídas.

\section{Agradecimentos}

A Coordenação de Aperfeiçoamento de Pessoal de Nível Superior (CAPES) pela bolsa concedida, e ao Instituto de Pesquisas Jardim Botânico do Rio de Janeiro, onde foi desenvolvida a pesquisa.

\section{Referências}

ANGIOSPERM PHYLOGENY GROUP III. An update of the Angiosperm Phylogeny Group classification for the orders and families of flowering plants: APG III. Botanical Journal of the 
Linnean Society, Londres, v. 161, p. 105-121, 2009.

ARAÚJO, R. S. et al. Aporte de serrapilheira e nutrientes ao solo em três modelos de revegetação na Reserva Biológica de Poço das Antas, Silva Jardim, RJ. Floresta e Ambiente, Seropédica, v. 12, n. 2, p. 15-21, 2006.

ARONSON, J.; DURIGAN, G.; BRANCALION, P. H. S. Conceitos e definições correlatos à ciência e a prática da restauração ecológica. Instituto Florestal Série Registros, São Paulo, n. 44, p. 1-38, 2011.

BAYLÃO JÚNIOR, H. F.; VALCARCEL, R.; NETTESHEIN, F. C. Fatores do meio físico associados ao estabelecimento de espécies rústicas em ecossistemas perturbados na Mata Atlântica, Piraí, RJ - Brasil. Ciência Florestal, Santa Maria, v. 23, n. 3, p. 305-315, 2013.

BOYD, C. S.; SVEJCAR, T. J. The influence of plant removal on succession in Wyoming big sagebrush. Journal of Arid Environments, London, v. 75, n. 8, p. 734-741, 2011.

BUENO, A.; LLAMBÍ, L. D. Facilitation and edge effects influence vegetation regeneration in old-fields at the tropical Andean forest line. Applied Vegetation Science, [s. l.], v. 18, n. 4, p. 613623, 2015.

CALLAWAY, R. M. Positive interactions among plants. The Botanical Review, New York, v. 61, n. 4, p. 306-349, 1995.

CHEUNG, K. C.; LIEBSCH, D.; MARQUES, M. C. M. Forest recovery in newly abandoned pastures in southern Brazil: implications for the Atlantic Rain Forest resilience. Natureza e Conservação, Curitiba, v. 8, n. 1, p. 66-70, 2010.

CHEUNG, K. C.; MARQUES, M. C. M.; LIEBSCH, D. Relação entre a presença de vegetação herbácea e a regeneração natural de espécies lenhosas em pastagens abandonadas na Floresta Ombrófila Densa do Sul do Brasil. Acta Botanica Brasilica, São Paulo, v. 23, n. 4, p. 1048-1056, 2009.

CONNELL, J. H.; SLATYER, R. O. Mechanisms of succession in natural communities and their role in community stability and organization. The American Naturalist, Chicago, v. 111, n. 982, p. 1119-1144, 1977.

CORTINES, E.; VALCARCEL, R. Influence of pioneer-species combinations on restoration of disturbed ecosystems in the Atlantic Forest, Rio de Janeiro, Brazil. Revista Árvore, Viçosa, MG, v. 33, n. 5, p. 925-934, 2009.

CUNHA, S. B.; FREITAS, M. W. D. Geossistemas e gestão ambiental na bacia hidrográfica do rio São João - RJ. GEOgraphia, Niterói, v. 6, n. 12, p. 87-110, 2004.

DERROIRE, G. et al. Isolated trees as nuclei of regeneration in tropical pastures: testing the importance of niche-based and landscape factors. Journal of Vegetation Science, Knivsta, v. 27, n. 4, p. 679-691, 2016.

DESIMONE, S. A. Balancing active and passive restoration in a nonchemical, research-based approach to coastal sage scrub restoration in Southern California. Ecological Restoration, Madison, v. 29, n. 1-2, p. 45-51, 2011.

DUARTE, L. S. et al. Testing for the influence of niche and neutral factors on sapling community assembly beneath isolated woody plants in grasslands. Journal of Vegetation Science, Knivsta, v. 21, n. 3, p. 462-471, 2010.

DUARTE, L. S. et al. What saplings can tell us about forest expansion over natural grasslands. Journal of Vegetation Science, Knivsta, v. 17, n. 6, p. 799-808, 2006.

FIGUEIREDO, P. H. et al. Germinação ex situ do banco de sementes do solo de capoeira em restauração florestal espontânea a partir do manejo do sombreamento. Scientia Forestalis, 
Piracicaba, v. 42, n. 101, p. 69-80, 2014.

GARBIN, M. L. et al. Subordinate, not dominant, woody species promote the diversity of climbing plants. Perspectives in Plant Ecology, Evolution and Systematics, Jena, v. 14, n. 4, p. 257-265, 2012.

GARCÍA-ORTH, X.; MARTÍNEZ-RAMOS, M. M. Isolated trees and grass removal improve performance of transplanted Trema micrantha (L.) Blume (Ulmaceae) saplings in tropical pastures. Restoration Ecology, Washington, v. 19, n. 1, p. 24-34, 2011.

GROENEVELD, J. et al. The impact of fragmentation and density regulation on forest succession in the Atlantic rain forest. Ecological Modelling, Amsterdam, v. 220, n. 1, p. 2450-2459, 2009.

GUEVARA, S. et al. The role of remnant forest trees in tropical secondary succession. Vegetatio, Dordrecht, v. 66, n. 2, p. 77-84, 1986.

HOLL, K. D. Effect of shrubs on tree seedling establishment in an abandoned tropical pasture. Journal of Ecology, London, v. 90, n. 1, p. 179-187, 2002.

HOLL, K. D.; AIDE, T. M. When and where to actively restore ecosystems? Forest Ecology and Management, Amsterdam, v. 261, n. 10, p. 1558-1563, 2011.

HOLL, K. D. et al. Tropical montane forest restoration in Costa Rica: overcoming barriers to dispersal and establishment. Restoration Ecology, Washington, v. 8, n. 4, p. 339-349, 2000.

KOLB, S. R. Islands of secondary vegetation in degraded pastures of Brazil: their role in reestablishing Atlantic Coastal Forest. 1993. Dissertation (Ph.D.) - University of Georgia, Athens, 1993.

LIRA, P. K. et al. Land-use and land-cover change in Atlantic Forest landscapes. Forest Ecology and Management, Amsterdam, v. 278, n. 1, p. 80-89, 2012.

MARTINS, C. R.; LEITE, L. L.; HARIDASAN, M. Capim-gordura (Melinis minutiflora P. Beauv.), uma gramínea exótica que compromete a recuperação de áreas degradadas em Unidades de Conservação. Revista Árvore, Viçosa, MG, v. 28, n. 5, p. 739-747, 2004.

MAZA-VILLALOBOS, S.; BALVANERA, P.; MARTÍNEZ-RAMOS, M. Early regeneration of tropical dry forest from abandoned pastures: contrasting chronosequence and dynamic approaches. Biotropica, Washington, v. 43, n. 6, p. 666-675, 2011.

MCINTIRE, E. J. B.; FAJARDO, A. Facilitation as a ubiquitous driver of biodiversity. New Phytologist, Cambridge, v. 201, n. 2, p. 403-416, 2014.

MIRANDA, C. C. et al. Avaliação das preferências ecológicas Clidemia urceolata DC. em ecossistemas perturbados. Revista Árvore, Viçosa, MG, v. 35, n. 5, p. 1135-1144, 2011.

MORAES, L. F. D. et al. Plantio de espécies arbóreas nativas para a restauração ecológica na Reserva Biológica de Poço das Antas, Rio de Janeiro, Brasil. Rodriguésia, Rio de Janeiro, v. 57, n. 3, p. 477-489, 2006.

MORO, M. F.; MARTINS, F. R. Métodos de levantamento do componente arbóreo - arbustivo. p. 174-212. In: FELFILI, J. M. et al. Fitossociologia no Brasil: métodos e estudo de casos. Viçosa, MG: Ed. UVF, 2011. v. 1.

MORRINSON, E. B.; LINDELL, C. A. Active or passive forest restoration? Assessing restoration alternatives with avian foraging behavior. Restoration Ecology, Washington, v. 19, n. 201, p. 170-177, 2010.

NEVES, G. M. S.; PEIXOTO, A. L. Florística e estrutura da comunidade arbustivo-arbórea de dois remanescentes em regeneração de Floresta Atlântica secundária na Reserva Biológica de Poço das Antas, Silva Jardim, Rio de Janeiro. Pesquisas Botânica, São Leopoldo, v. 59, p. 71-112, 
2008.

OKSANEN, J. et al. Vegan: community ecology package. R package version 2.0-9. [S. l: s. n.], 2013. Disponível em: http://CRAN.R-project.org/package=vegan.

PADILLA, F. M.; PUGNAIRE, F. I. The role of nurse plants in the restoration of degraded environments. Frontiers in Ecology and the Environment, Washington, v. 4, n. 4, p. 196-202, 2006.

R DEVELOPMENT CORE TEAM. R: a language and environment for statistical computing. Vienna: R Foundation for Statistical Computing, 2013. Disponível em: http://www.r-project.org/.

REIS, A. et al. Nucleation in tropical ecological restoration. Scientia Agricola, Piracicaba, v. 67, n. 2, p. 244-250, 2010.

REIS, A. et al. Restauração de áreas degradadas: a nucleação como base para incrementar os processos sucessionais. Natureza e Conservação, Curitiba, v. 1, n. 1, p. 28-36, 2003.

RIBEIRO, M. C. et al. The Brazilian Atlantic Forest: how much is left, and how is the remaining forest distributed? Implications for conservation. Biological Conservation, Essex, v. 142, n. 6, p. 1141-1153, 2009.

ROBERTS, D. W. Labdsv: ordination and multivariate analysis for ecology. R package version 1.6-1. [S. l: s. n.], 2013. Disponível em: http://CRAN.R-project.org/package=labdsv.

SÁNCHEZ-TAPIA, A. Regeneração natural e restauração ecológica em capoeiras antropizadas de Mata Atlântica submetidas a queimadas. 2011. Dissertação (Mestrado em Ecologia) Universidade Federal do Rio de Janeiro, Rio de Janeiro, 2011.

SANDOR, M. E.; CHAZDON, R. L. Remnant trees affect species composition but not structure of tropical second-growth forest. PLoS ONE, [s. l.], v. 9, n. 1, p. e83284. doi: 10.1371/journal. pone.0083284, 2014.

SANSEVERO, J. B. B. et al. Natural regeneration in plantations of native trees in lowland Brazilian Atlantic Forest: community structure, diversity, and dispersal syndromes. Restoration Ecology, Washington, v. 19, n. 3, p. 379-389, 2011.

SANTOS, J. F. et al. Horizontal structure and floristic composition of the shrubby-arboreal strata in forest planted to rehabilitate a degraded area of the Brazilian Atlantic Forest, Rio de Janeiro. Ciencia y Investigación Agraria, Santiago, v. 38, n. 1, p. 95-106, 2011.

SCARANO, F. R.; CEOTTO, P. Brazilian Atlantic forest: impact, vulnerability, and adaptation to climate change. Biodiversity and Conservation, Dordrecht, v. 24, p. 2319-2331, 2015.

SCOWCROFT, P. G.; YEH, J. T. Passive restoration augments active restoration in deforested landscapes: The role of root suckering adjacent to planted stands of Acacia koa. Forest Ecology and Management, Amsterdam, v. 305, p. 138-145, 2013.

SHONO, K.; CADAWENG, E. A.; DURST, P. B. Application of assisted natural regeneration to restore degraded tropical forestlands. Restoration Ecology, Washington, v. 15, n. 4, p. 620-626, 2007.

SMITH, R. L. Ecology and field biology. $5^{\text {th }}$ ed. New York: Harper Collins College Publishers, 1996. $740 \mathrm{p}$.

SOCIETY FOR ECOLOGICAL RESTORATION INTERNATIONAL. Princípios da SER International sobre a restauração ecológica. Grupo de Trabalho sobre Ciência e Política versão 2. Tucson: SER, 2004. Disponível em: www.ser.org.

STACHOWICZ, J. J. Mutualism, facilitation and the structure of ecological communities. 
BioScience, Washington, v. 51, n. 3, p. 235-246, 2001.

SWAINE, M. D.; WHITMORE, T. C. On the definition of ecological species groups in tropical rain forests. Vegetatio, Dordrecht, v. 75, n. 1/2, p. 81-86, 1988.

WESTMAN, W. E. Measuring the Inertia and Resilience of Ecosystems. BioScience, Washington, v. 28, n.11, p. $705-710,1978$.

YARRANTON, G. A.; MORRISON, R. G. Spatial dynamics of a primary succession: nucleation. Journal of Ecology, London, v. 62, n. 2, p. 417-428, 1974.

ZAHAWI, R. A.; AUGSPURGER, C. K. Early plant succession in abandoned pastures in Ecuador. Biotropica, Hoboken, v. 31, n. 4, p. 540-552, 1999.

ZAHAWI, R. A.; AUGSPURGER, C. K. Tropical forest restoration: tree islands as recruitment foci in degraded lands of Honduras. Ecological Applications, Washington, v. 16, n. 2, p. 464-478, 2006. 